\title{
Análisis de la variación de temperatura en un pozo geotérmico a través de pruebas de TRT y enfriamiento en la ciudad de Talca, Chile
}

Analysis of temperature variation in a geothermal borehole through TRT and cooling tests in the city of Talca, Chile

Fecha de entrega: 4 de noviembre 2019

Fecha de aceptación: 5 de marzo 2020

\section{Fernanda Ochoa ${ }^{1}$, David Zamora-Barraza ${ }^{2}$, Walter Schmidt ${ }^{2}$, Dante Figueroa $^{1}$ y Arturo Belmonte ${ }^{1}$}

${ }^{1}$ Departamento de Geofísica, Universidad de Concepción, Avda. Esteban Iturra s/n - Barrio Universitario, Casilla 160-C, Concepción, Chile, fernandaochoa.esp@gmail.com,dantefigueroa@udec.cl, abelmonte@dgeo.udec.cl

${ }^{2}$ Departamento de Obras Civiles, Universidad Católica del Maule, Campus San Miguel, Avenida San Miguel 3605, Casilla 617, Talca, Chile,dzamora@ucm.cl,wschmidt1989@gmail.com

En el campus San Miguel de la Universidad Católica del Maule en la ciudad de Talca (Chile), se analizaron la conductividad y resistencia térmica de un pozo geotérmico por medio de un test de respuesta térmica (TRT) en función de tiempos de descarte (TD) para 3 pruebas de inyección de calor diferentes, resultando la conductividad térmica de, al menos, $1.6 \mathrm{~W}$ $m^{-1} K^{-1}$ y una resistencia térmica de $0.25 \mathrm{mK} W^{-1}$ (valores más desfavorables). Aprovechando el proceso de calentamiento a través del TRT, se analizó la capacidad de enfriamiento del intercambiador de calor geotérmico vertical desde un punto de vista teórico y práctico. Se obtuvieron perfiles de temperatura a lo largo del pozo en diferentes intervalos de tiempo por medio de un sensor de presión de agua y temperatura, y de esta forma, se obtuvo una curva de temperatura promedio del pozo hasta recuperar la temperatura de equilibrio. Esta última se comparó con la curva teórica del proceso de enfriamiento. Los resultados indican que las curvas de enfriamiento obtenidas de forma práctica son similares a las curvas teóricas. Junto con esto, se obtuvo un valor para la conductividad térmica de $2.35 \mathrm{Wm}^{-1} \mathrm{~K}^{-1}$.

Palabras clave: pozo geotermal, temperatura, conductividad térmica, borehole geothermal heat exchanger (BHE), test de respuesta térmica (TRT), prueba de enfriamiento
On the San Miguel campus of the Catholic University of Maule, in the city of Talca (Chile), the conductivity and thermal resistance of a geothermal borehole were analyzed through a thermal response test (TRT) based on initial ignoring time (IIT) for 3 different heat injection tests, resulting in the thermal conductivity of at least 1.6 $W^{-1} \mathrm{~K}^{-1}$ and a thermal resistance of $0.25 \mathrm{mKW^{-1 }}$ (most unfavorable values). Taking advantage of the heating process through the TRT, the cooling capacity of the vertical geothermal heat exchanger was analyzed from a theoretical and practical point of view. Temperature profiles were obtained along the borehole at different time intervals through a water pressure and temperature sensor, and in this way, an average temperature curve of the borehole until the equilibrium temperature was obtained. The latter was compared with the theoretical curve of the cooling process. The results indicate that the cooling curves obtained in a practical way are similar to the theoretical curves. Together with this, a value for the thermal conductivity of $2.35 \mathrm{Wm}^{-1} \mathrm{~K}^{-1}$ was obtained.

Keywords: geothermal borehole, temperature, thermal conductivity, borehole geothermal heat exchanger (BHE), thermal response test (TRT), cooling test

\section{Introducción}

Entre los tipos de energías más eficientes en climatización de espacios se encuentra la energía geotérmica de baja entalpía. Esta forma de energía aprovecha la inercia térmica del subsuelo, el cual mantiene una temperatura entre 5 y $30^{\circ} \mathrm{C}$ dependiendo de la latitud y de las condiciones geoquímicas presentes en el terreno (capacidad térmica de los minerales, presencia de agua estática o en movimiento en el acuífero, permeabilidad del suelo) y llega a profundidades máximas de 400 m (Llopis y Rodrigo, 2008). Este sistema de climatización se basa en una bomba de calor geotérmica 
(BCG) conectada a tubos intercambiadores de calor por los que fluye un líquido portador de calor (agua o agua con anticongelante) que cede o absorbe calor hacia o desde el subsuelo, según los requerimientos de climatización. Este calor es transportado posteriormente por un sistema de distribución al interior de la edificación.

Los intercambiadores de calor pueden ser horizontales o verticales. Los experimentos y resultados aquí presentados se han realizado en un contexto de intercambiador de calor vertical, por lo que nos referimos a éste.

Los intercambiadores verticales consisten en uno o varios tubos enterrados en pozos de profundidad variable. Existe una distinción entre sistema abierto y cerrado de intercambiador vertical. En los abiertos, el agua subterránea que contiene el subsuelo se extrae del pozo a través de un solo tubo. Después de enfriar o calentar el agua en una bomba de calor, el agua es reinyectada al pozo. La principal ventaja de este sistema es que el fluido portador de calor está en contacto directo con las rocas del pozo, por lo que existe una excelente transferencia de calor. Sin embargo, se requieren ciertas condiciones hidrogeológicas y geoquímicas favorables para la construcción de este tipo de pozo y evitar la contaminación o agotamiento de la fuente. Además, la temperatura de extracción debe ser superior a $0^{\circ} \mathrm{C}$ para evitar el congelamiento. Los principales tipos de intercambiadores geotérmicos se pueden ver en la Figura 1.

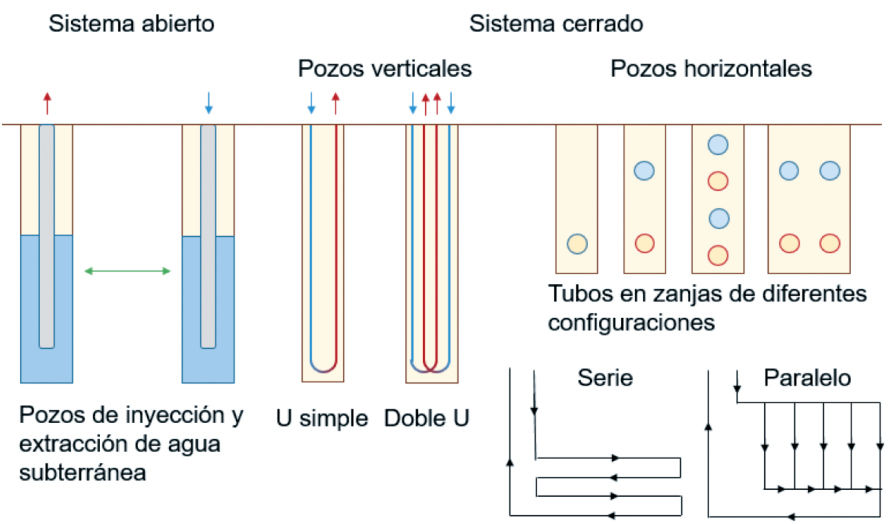

Figura 1: Tipos de intercambiadores geotérmicos

Cuando se inserta uno o más tubos en forma de $\mathrm{U}$ se obtiene un sistema cerrado. El fluido portador de calor que circula a través del sistema está completamente separado del medio circundante, haciendo posible usar otros tipos de fluido como agua con anticongelantes y trabajar con temperaturas menores a $0^{\circ} \mathrm{C}$. La transferencia de calor es inferior al del sistema abierto debido a las resistencias térmicas adicionales entre la pared de la tubería, el relleno del pozo (mortero utilizado para dar estabilidad al pozo y una superficie de contacto intercambiador-suelo, por ejemplo: mezcla de arena, cemento y bentonita) y el suelo circundante, lo que reduce la capacidad del sistema. Los tubos de los sistemas cerrados son generalmente de polietileno de alta densidad (HDPE) en forma de U o doble $\mathrm{U}$, enterrados en uno o varios pozos, de 50 a $200 \mathrm{~m}$ de profundidad.

Los sistemas verticales cerrados aprovechan el calor del subsuelo a gran profundidad. En los primeros $15 \mathrm{~m} \mathrm{la}$ temperatura es alterada por las condiciones meteorológicas en mayor medida, pero a partir de los $15 \mathrm{~m}$ la temperatura permanece constante y desde los $20 \mathrm{~m}$ en adelante aumenta en $3^{\circ} \mathrm{C}$ cada $100 \mathrm{~m}$ (Jara y Martínez, 2016). Esta condición le permite realizar al sistema una mayor transferencia de calor por metro lineal de tubo que en el caso horizontal, llegando a entregar entre 32 y $43 \mathrm{~W}$ por metro perforado (Natural Resources Canada, 2004), respecto de 20-29 $\mathrm{W} / \mathrm{m}$. La profundidad exacta de los pozos depende de la superficie a climatizar y la cantidad de perforaciones que se realicen.

La ventaja principal de estos sistemas es la superficie mínima de terreno libre requerido para su instalación y poder ser implementado tanto en construcciones existentes como en ejecución. Además, el líquido portador de calor se encuentra libre de minerales o partículas que puedan disminuir la vida útil de la instalación y no perturba las aguas subterráneas que puede haber cerca del pozo, contrario a los sistemas abiertos, por lo que el sistema es independiente de la presencia de aguas subterráneas. Su realización, sin embargo, implica un alto costo de inversión, ya que requiere perforaciones a mayor profundidad y de equipos especiales para efectuarlas (Jara y Martínez, 2016).

\section{Objetivos}

Conocer el comportamiento de un pozo intercambiador de calor vertical (BHE: borehole geothermal heat exchanger), estimar el desempeño durante su vida útil y controlar los efectos sobre el medio son elementos esenciales para un adecuado diseño de sistemas geotérmicos. Para esto, se 
requiere conocer las propiedades térmicas del suelo, como la temperatura del suelo inalterado, la conductividad térmica, la difusividad térmica y la resistencia térmica del pozo.

En el presente trabajo, una serie de modelos analíticos y numéricos se han desarrollado para simular el comportamiento de los intercambiadores de calor en régimen transitorio, utilizando modelos de fuente lineal y cilíndrica. Dicho análisis se concentra en 2 fases de análisis: una fase de calentamiento y otra de enfriamiento del pozo de estudio. Ambas fases nos permiten hacer determinaciones de los parámetros de interés.

El sitio de estudio corresponde a un pozo geotérmico perteneciente a un sistema de 10 pozos construidos el año 2011 en el campus San Miguel de la Universidad Católica del Maule en la ciudad de Talca, Chile (35 26' 09.07''S, $71^{\circ} 37^{\prime} 11.02$ ' O).

\section{Metodología}

Para conocer las propiedades térmicas del suelo en instalaciones geotérmica verticales tipo cerradas con bomba de calor se usa actualmente el denominado test de respuesta térmica (TRT). Este consiste en hacer circular por el pozo una carga térmica conocida (potencia) y registrar las temperaturas de entrada y salida del fluido circulante (por ejemplo, agua).

La respuesta térmica del pozo es el cambio que ocurre en su temperatura cuando el fluido portador de calor fluye a través de los intercambiadores de calor por un cierto periodo de tiempo (Monzó, 2011). El estudio de la temperatura del fluido con respecto al tiempo permite la estimación de las propiedades térmicas alrededor del pozo.

Los principales modelos analíticos usados para evaluar los datos de respuesta térmica son los modelos de fuente lineal y fuente cilíndrica. Mogensen (1983) fue el primero en aplicar el modelo de fuente lineal para estimar la conductividad térmica del suelo a través de datos experimentales. Este modelo se ocupa por su simplicidad. La temperatura del suelo puede ser escrita en función del tiempo $t$ y el radio $r$ alrededor de una fuente lineal con una tasa constante de inyección de calor (calor $q$ por unidad de longitud) a través de una línea que va en el eje vertical del pozo inserto en un medio sólido infinito (Carslaw y Jaeger, 1959).

El modelo supone para el TRT que la transferencia de calor es sólo por conducción y en sentido radial, despreciando la convección y los flujos verticales (Sharqawy et al., 2009). Las temperaturas de entrada y salida de los pozos intercambiadores de calor, el caudal y la potencia introducida son registradas durante la prueba que dura comúnmente entre 2 y 4 días.

Aplicando una aproximación planteada por del Valle (2012), sujeta en términos prácticos a considerar periodos de muestreo superiores a cierto umbral $\left(\alpha t / r^{2} \geq 20\right)$ para mantener errores en el orden del $2 \%$, se puede demostrar que la temperatura del fluido en la tubería $T(r, t)$ se puede describir de la siguiente forma:

$$
\mathrm{T}_{\mathrm{f}}(t)=\frac{q_{\mathrm{c}}}{4 \pi \lambda} \ln (t)+q_{\mathrm{c}}\left[\mathrm{R}_{\mathrm{b}}+\frac{1}{4 \pi \lambda}\left(\ln \left[\frac{4 \alpha}{r^{2}}\right]-\gamma\right)\right]+\mathrm{T}_{0}
$$

donde $q_{\mathrm{c}}$ es el calor por unidad de longitud (W/m), $\mathrm{T}_{\mathrm{f}}(\mathrm{t})$ es la temperatura del fluido en la tubería $\left({ }^{\circ} \mathrm{C}\right), \mathrm{R}_{\mathrm{b}}$ es la resistencia térmica del pozo $\left(\mathrm{mKW}^{-1}\right), \lambda$ es la conductividad térmica del sistema $\left(\mathrm{Wm}^{-1} \mathrm{~K}^{-1}\right), \alpha$ es la difusividad térmica del terreno $\left(=9.6 \cdot 10^{-7} \mathrm{~m}^{2} / \mathrm{s}\right), \gamma$ es la constante de Euler $(=$ 0.5772), $\mathrm{T}_{0}$ es la temperatura inalterada del terreno y $r$ es el radio del pozo. La ecuación (1) presenta una relación lineal con respecto al tiempo en escala logarítmica, siendo $m$ la pendiente y $c$ el valor de temperatura cuando el tiempo es igual a cero y conocido como el valor de la ordenada en el origen.

$$
\begin{aligned}
& \mathrm{T}_{\mathrm{f}}(t)=m \ln (t)+c \\
& m=\frac{q_{\mathrm{c}}}{4 \pi \lambda} \\
& c=q_{c}\left[\mathrm{R}_{\mathrm{b}}+\frac{1}{4 \pi \lambda}\left(\ln \left[\frac{4 \alpha}{r^{2}}\right]-\gamma\right)\right]+\mathrm{T}_{0}
\end{aligned}
$$

De esta forma, si medimos la evolución de las temperaturas durante un determinado transcurso de tiempo, podemos construir la relación lineal (2). Dado el valor de $q$ (dato), podemos desde la relación (3) estimar la conductividad térmica efectiva del sistema $(\lambda)$, y usando la relación (4), calcular la resistencia térmica del pozo $\left(\mathrm{R}_{\mathrm{b}}\right)$.

El proceso de enfriamiento toma lugar con posterioridad al "apagado" del sistema TRT, mientras que el perfil de temperaturas del "suelo inalterado" se realizó llenando las tuberías con agua, y luego de 2 semanas de reposo, se midió la temperatura del pozo. 


\section{Lugar de mediciones y equipamiento}

El intercambiador vertical geotérmico estudiado está ubicado en el edificio Parque Tecnológico de la Universidad Católica del Maule UCM, en Talca. Este edificio forma parte del Proyecto de aprovechamiento geotérmico de baja entalpía en edificios de usos múltiples: Parque Tecnológico de la UCM (Castro et al., 2012). El pozo en estudio es uno de los 10 pozos geotérmicos con los que cuenta la instalación, con profundidades variables entre 60 y $80 \mathrm{~m}$ sobre suelo arenoso saturado (tipo de suelo predominante en el lugar, de acuerdo a Oyarce (2018)) con difusividad térmica estimada en $9.6 \cdot 10^{-7} \mathrm{~m}^{2} / \mathrm{s}$ de acuerdo a la norma VDI 4640 (2010) para arenas saturadas. La perforación posee un diámetro de $140 \mathrm{~mm}$ y $69 \mathrm{~m}$ de profundidad; los tubos tienen una configuración de doble $\mathrm{U}$, modelo RAUGEO PE-Xa, de $32 \mathrm{~mm}$ de diámetro, $3 \mathrm{~mm}$ de espesor y un espaciamiento entre tubos de $60 \mathrm{~mm}$. Las tuberías se mantienen verticales desde los $2 \mathrm{~m}$ de profundidad, previo a esto, las tuberías se redistribuyen de manera horizontal para conectarse a la sala de máquinas.

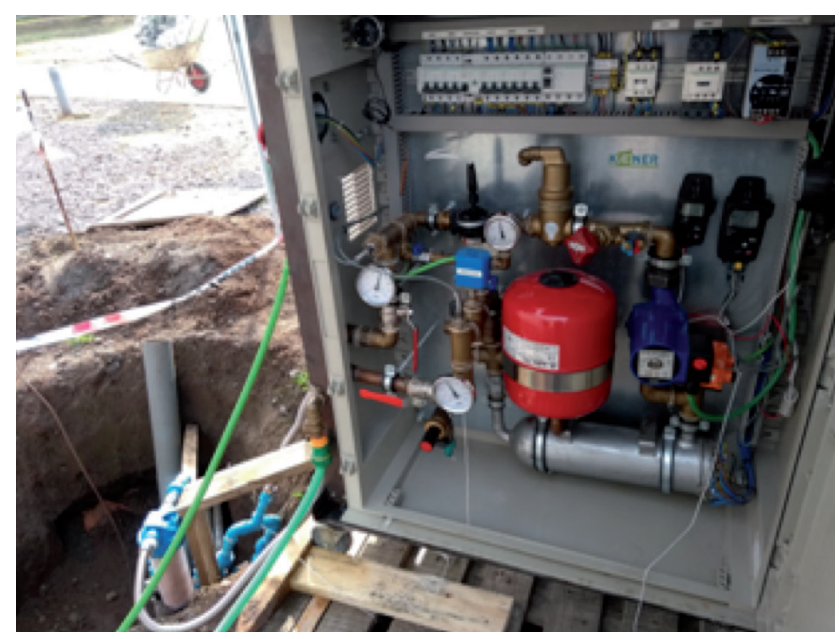

Figura 2: Sistema de test de respuesta térmica (TRT).

El sistema TRT, mostrado en la Figura 2, cuenta con una bomba de agua de 11 a 13 lt/min de caudal, un calefactor de agua de $5 \mathrm{~kW}$ de potencia y tubos de entrada y retorno al pozo. Un sensor de temperatura Testo $177-\mathrm{T} 4$, de $+/-0.3^{\circ} \mathrm{C}$ de error, registra y almacena la temperatura ambiente y la temperatura de entrada y retorno del fluido, mientras que el caudal instantáneo del sistema es registrado por un sensor Testo 175-S2.

Para determinar los perfiles de temperatura del pozo, se utilizó un dispositivo de registro de temperatura y presión de agua, introduciéndolo en el pozo en enfriamiento a intervalos de $1 \mathrm{~min}$ cada $2 \mathrm{~m}$ de profundidad, tiempo necesario para estabilizar el instrumento y el flujo de agua interno (se asume que la temperatura registrada es la temperatura del suelo a tal profundidad).

\section{Resultados y análisis Temperatura del terreno no perturbado}

Con el objetivo de conocer la temperatura del terreno no perturbado y así contar con una temperatura inicial del pozo para el posterior ensayo del TRT, se midió la temperatura a través de un proceso de inmersión de una sonda con un sensor de temperatura.

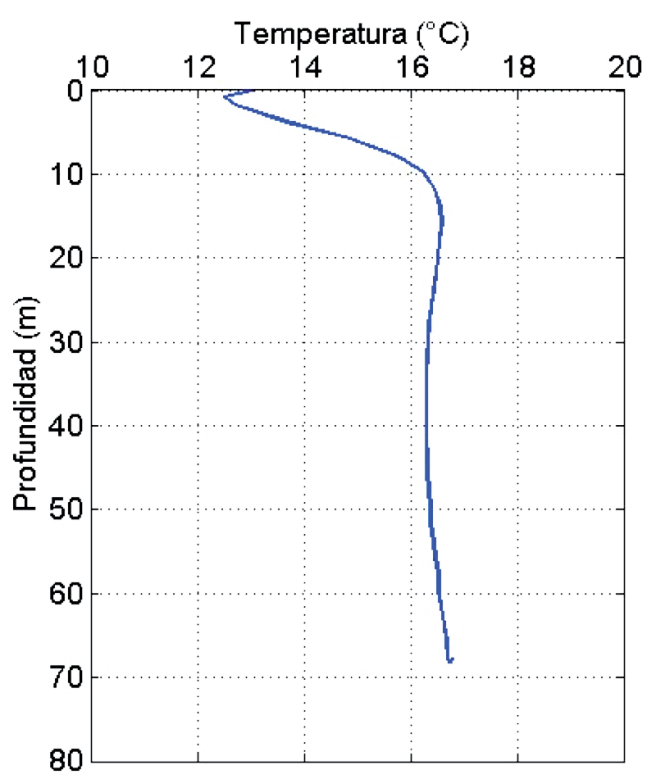

Figura 3: Perfil de temperaturas del pozo inalterado. Mediciones en inmersión realizadas con sonda

En la Figura 3 se muestra el perfil de temperaturas del suelo no perturbado en función de la profundidad. Durante la inmersión del sensor de temperatura; ésta varía ampliamente en los primeros $14 \mathrm{~m}$ en un rango de 11.5 a $16.5^{\circ} \mathrm{C}$ debido a la influencia de las condiciones atmosféricas y a los ciclos diarios, pero a mayor profundidad la temperatura se muestra relativamente estable, con un ligero aumento gradual de temperatura a partir de $\operatorname{los} 40 \mathrm{~m}$. La temperatura promedio del pozo es de $16.4^{\circ} \mathrm{C}$, considerada desde los 14 hasta $\operatorname{los} 69 \mathrm{~m}$ de profundidad, rango en que la temperatura del terreno no se ve influenciada por las condiciones ambientales externas. 
Conductividad térmica del terreno y resistencia térmica del pozo

Se realizaron 3 ensayos durante el año 2018 para determinar la conductividad térmica del terreno, cada uno de distinta duración. El primero (Test 1) se realizó entre los días 17 y 21 de agosto y alcanzó una duración de 93 hrs y 1 min; el segundo (Test 2) durante los días 3 y 6 de septiembre y tuvo una duración de 68 hrs y 9 min; el tercero (Test 3) se realizó los días 10 y 11 de septiembre, alcanzando una duración de 24 hrs.

La Figura 4 muestra la evolución de la temperatura media del pozo en función del tiempo (horas transcurridas desde inicio de cada operación) para las 3 pruebas, mientras que en la Figura 5 se muestra la evolución de la temperatura en función del tiempo en escala logarítmica. En la Figura 4 se puede apreciar como la temperatura crece rápidamente durante las primeras $10 \mathrm{hrs}$ aproximadamente, para luego mostrar una tendencia a una temperatura máxima y constante que, en el caso del Test 1 , con el mayor periodo de observación, alcanza los $38.9^{\circ} \mathrm{C}$. En la Figura 5 se puede observar la clara tendencia de linealidad predicha por la ecuación (2) a partir de los primeros 45 min de cada ensayo. Considerando estos valores de temperatura como variables $\mathrm{T}_{\mathrm{f}}(t)$, es a partir de las ecuaciones (3) y (4) que, determinando los parámetros $m$ y $c$ de la ecuación (2), podemos estimar valores para la conductividad térmica del sistema $\lambda$ y la resistividad térmica del pozo $R_{b}$. Esta determinación se realiza aplicando el método de regresión lineal por mínimos cuadrados, para un rango específico de tiempo. El tiempo mínimo está condicionado por la ecuación (1), y que, en este caso, implica que debe ser mayor o igual a $28 \mathrm{hrs}$. A este límite inferior se le conoce

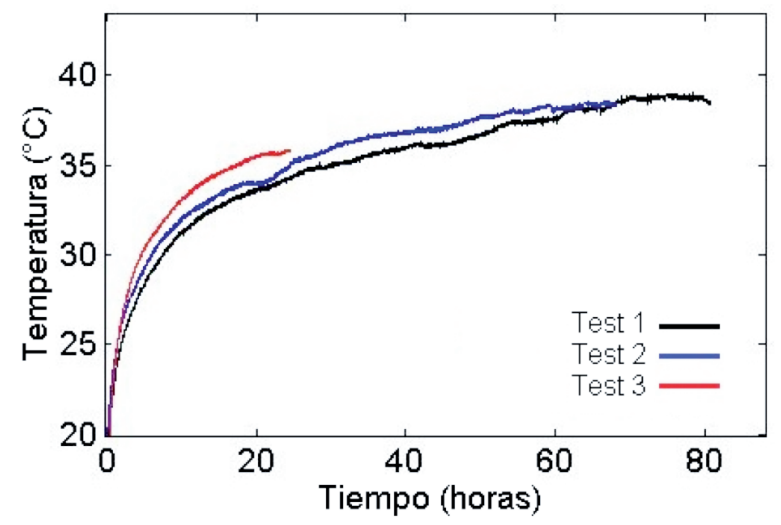

Figura 4: Temperatura del pozo en función del tiempo.

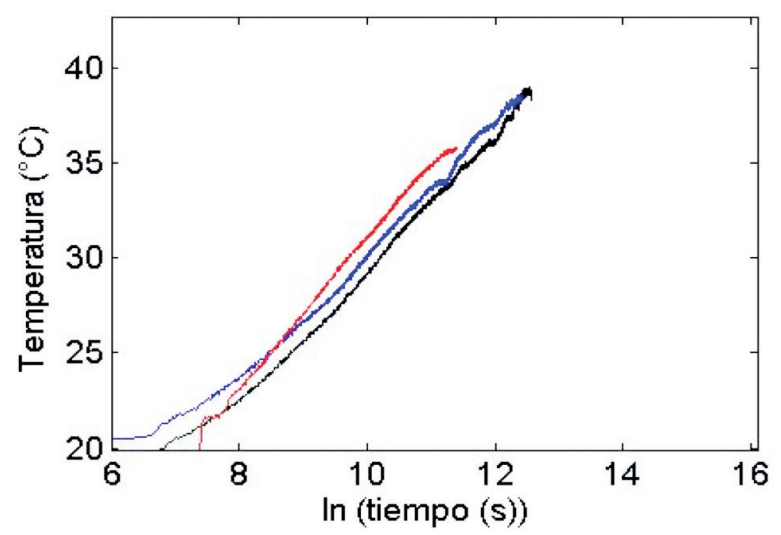

Figura 5: Temperatura del pozo en función del tiempo en escala logarítmica. El significado del color de las líneas es similar al de la Figura 4.

como Tiempo de Descarte TD.

Para dilucidar un valor estadísticamente representativo, observamos que, si hacemos variar el tiempo de descarte desde el mínimo hasta el final de cada serie de datos, el número de observaciones disminuye linealmente hasta llegar a cero. Para evitar esto y sus consecuencias en la estimación de parámetros de error y control de la regresión (coeficiente de correlación $\mathrm{R}^{2}$ ), obligamos a realizar los cálculos para periodos de tiempo donde el número de observaciones sea similar. Esto se traduce en aplicar un periodo de análisis móvil de $20 \mathrm{hrs}(\Delta \tau=20 \mathrm{hrs})$, es decir, la estimación de $m$ y $c$ la hacemos entre el Tiempo de Descarte, que parte en las $28 \mathrm{hrs,} \mathrm{y} \mathrm{un} \mathrm{tiempo} \mathrm{máximo} \mathrm{que}$ es igual a TD más 20 hrs. Hacer crecer este valor es posible y los resultados no cambian de manera relevante, pero se pierde la calidad de la regresión mucho antes. Con este rango específico de observación (20 hrs), las estimaciones parecen razonables hasta aproximadamente las $60 \mathrm{hrs}$ de experimento, para el Test 1, y hasta las $50 \mathrm{hrs}$ para el Test 2. El Test 3 queda fuera de este análisis debido a su corto periodo de observación. En la Figura 6 se muestra el comportamiento del coeficiente de correlación $\mathrm{R}^{2}$, desde donde es posible deducir esto si exigimos que $\mathrm{R}^{2}>0.85$.

Aplicando (3) y habiendo estimado el valor de la pendiente $m$ en (2), es posible estimar valores de conductividad térmica $\lambda$ para cada valor del tiempo de descarte TD. En la Figura 7 se muestran estos resultados. Como se puede apreciar, los valores de conductividad son variables. Si consideramos válida la idea de exigir un mayor tiempo de medición y además un valor de correlación alto, nos 


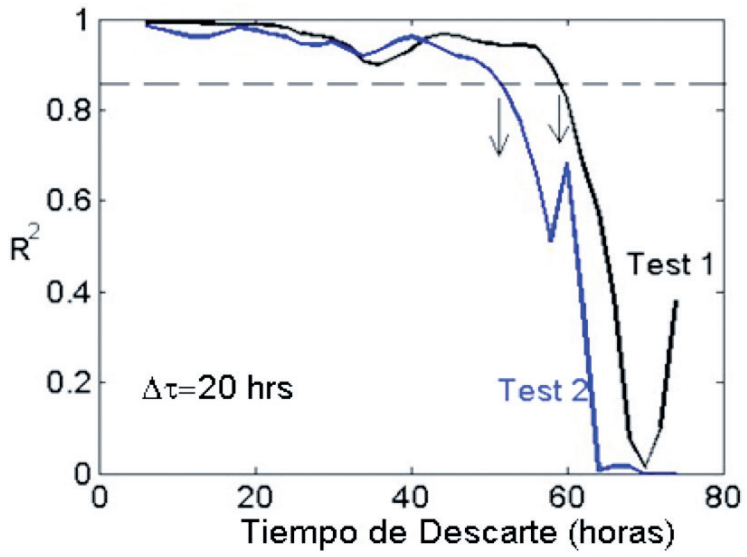

Figura 6: Coeficiente de correlación $\mathrm{R}^{2}$ en función del tiempo de realización experimental de los Tests números 1 y 2 .

acercamos al rango de valores de tiempo de descarte entre 50 (Test 2) y 60 hrs (Test 1). A valores mayores de TD, la disminución de las observaciones, así como la variabilidad de los valores de temperatura cerca del valor máximo, hacen que los valores calculados presenten una alta distorsión y su interpretación carezca de sentido. De esta forma el rango de valores plausibles para la conductividad térmica es 1.6 a $2.5 \mathrm{Wm}^{-1} \mathrm{~K}^{-1}$. De manera similar se puede mostrar que los valores de resistividad térmica $R_{b}$ son del orden de $0.25 \pm 0.03 \mathrm{mKW}^{-1}$.

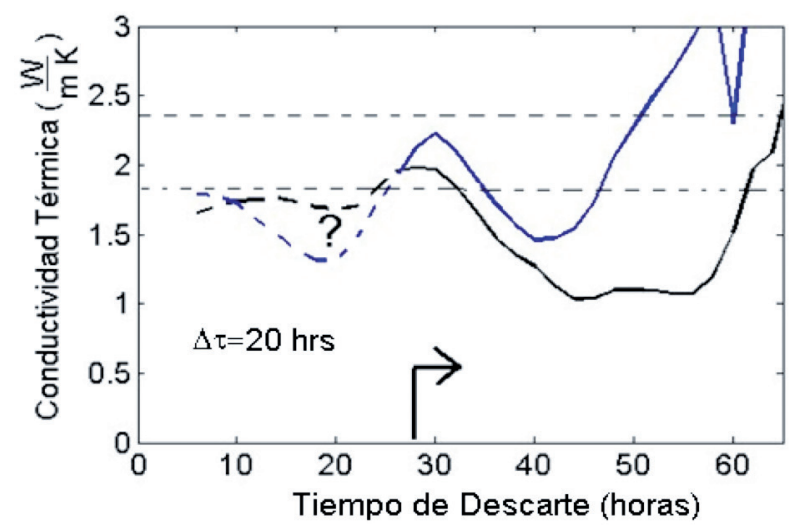

Figura 7: Conductividad térmica $\lambda$ en función del tiempo de realización experimental de los Tests números 1 y 2. El significado del color de las líneas es similar al de la Figura 6.

Aunque se puede esperar que un aumento en la duración de las pruebas de TRT entregue una estimación más precisa de las propiedades térmicas del terreno, y así considerar que los valores estimados de conductividad $\lambda$ son más acertados (Ingersoll et al., 1954), en el presente experimento se evidencia que el periodo de tiempo considerado para el análisis de regresión lineal es una variable poco sensible a variaciones de $\lambda$. Sin embargo, a partir de cierto tiempo de descarte (TD $=60-65 \mathrm{hrs}$ ), los resultados muestran una alta distorsión con una tendencia al aumento de $\lambda$, más una razón de la inestabilidad que la medición de temperatura parece tener cerca de su máximo permitido por el sistema y el terreno. Junto a esto, queda abierta la pregunta respecto a la incidencia que tiene la realización de más de una prueba de TRT en el terreno. Se observa que desde el Test 1 al 3 , la temperatura aumenta con mayor rapidez durante las primeras horas del experimento, incidiendo en que $\lambda$ tienda a decrecer durante el experimento, pero aparentemente al alcanzar el máximo de temperatura, en términos relativos, $\lambda$ tiende a crecer. No es claro a qué se debe esto. En esta línea, Lee et al. (2018) sugieren que a medida que la cantidad de calor que se 'inyecta' se incrementa, el valor de $\lambda$ deja de depender de TD.

De los presentes resultados, es posible constatar que una buena estimación de $\lambda$ parece requerir la condición de limitar el tiempo de descarte $(\mathrm{TD}=50-60 \mathrm{hrs}) \mathrm{y}$ definir una ventana de tiempo de análisis que asegure una cantidad de datos razonable, y por tanto, un análisis estadístico comparable, para cada valor de TD que se evalúa. Sin embargo, no es posible precisar con exactitud un valor para $\lambda$, sólo un rango amplio de posibles valores coincidentes con los esperados de 1.6 a $2.5 \mathrm{Wm}^{-1} \mathrm{~K}^{-1}$. En el caso, de la resistividad térmica $\mathrm{R}_{\mathrm{b}}$ ocurre la misma condición, pudiendo sí ajustar un valor promedio cercano a $0.25 \mathrm{mKW}^{-1}$.

\section{Curva de enfriamiento del pozo}

Con posterioridad al apagado de cada medición de TRT, se procedió a medir la temperatura en el pozo geotérmico en diferentes momentos y a distintas profundidades a medida que la temperatura disminuye hasta alcanzar la temperatura de equilibrio del terreno como se muestra en la Figura 8.

Con el objeto de analizar el conjunto de temperaturas más representativo del terreno, el análisis se concentra para el rango de profundidades entre 14 y $69 \mathrm{~m}$. Para cada instante de medición, se promedió el valor de las temperaturas obtenidas en el rango de profundidad señalado. En la Figura 9 se muestran estos resultados. Los círculos indican el dato de temperatura observado (cada color se refiere al número del test) y las curvas son el resultado del ajuste de la regresión lineal realizada siguiendo los pasos indicados anteriormente. 


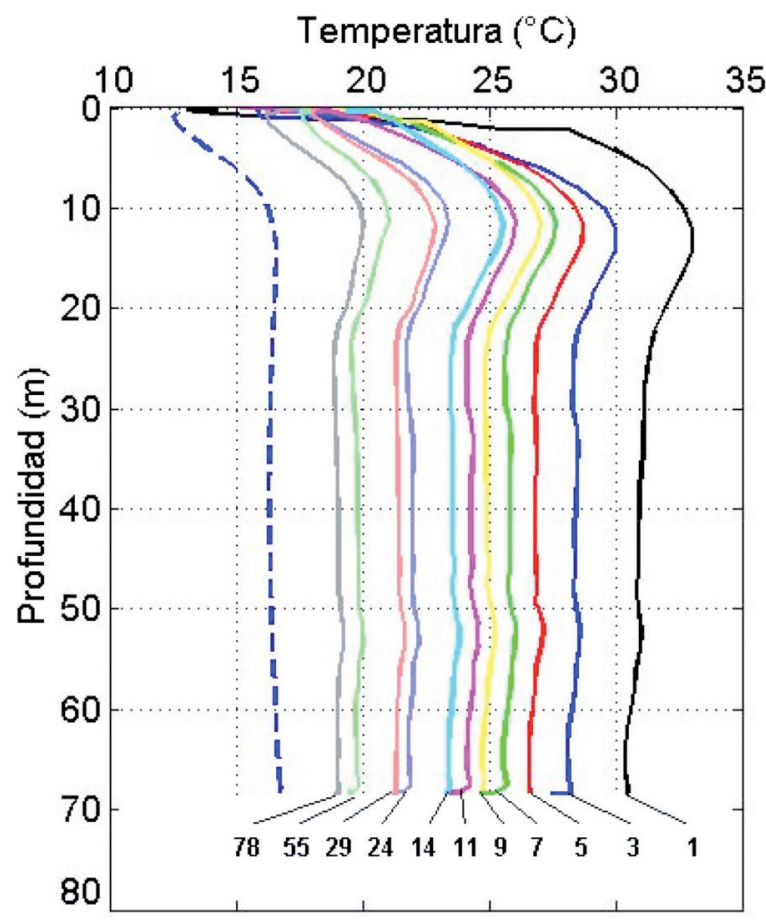

Figura 8: Perfiles de temperatura en profundidad obtenidos con el sensor de temperatura durante el ensayo 1. Los números en la parte baja y relacionada con cada curva corresponden al tiempo transcurrido en hrs desde que fue apagado el TRT. Como referencia, la curva de color azul entre-cortada corresponde a las variaciones de temperatura con la profundidad para el caso de un terreno no perturbado (referencia Figura 3).

Al comparar las curvas de enfriamiento de los Test 1, 2 y Test 3, se aprecia que la disminución del valor absoluto de la pendiente de este último podría relacionarse con que su temperatura inicial es menor, o bien, se puede decir que el aumento de la temperatura inicial incide en un valor absoluto de la pendiente mayor. Esto, si es cierto, podría tener su causa en el gradiente térmico existente entre el pozo y el terreno circundante: a mayor gradiente térmico, mayor valor de la pendiente $m$.

\section{Curva teórica del proceso de enfriamiento del pozo}

Los datos asociados al proceso de enfriamiento permiten plantearnos la posibilidad de proponer un modelo teórico que represente dichas observaciones y que dependa de las propiedades térmicas de los materiales involucrados, su geometría y las fórmulas de transferencia de calor planteadas anteriormente. Para esto, aplicamos la ley de conducción del calor de Fourier en un sistema conformado por distintos materiales concéntricos entorno a un eje de largo determinado. Se puede demostrar que se cumple: a)

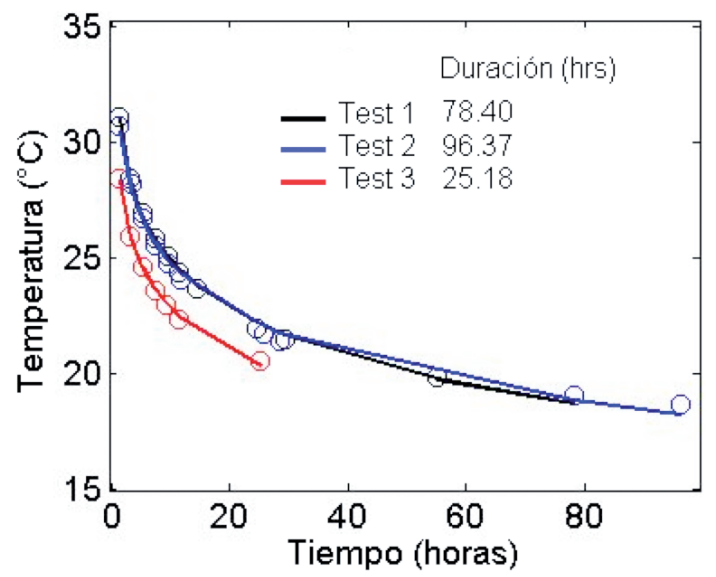

b)

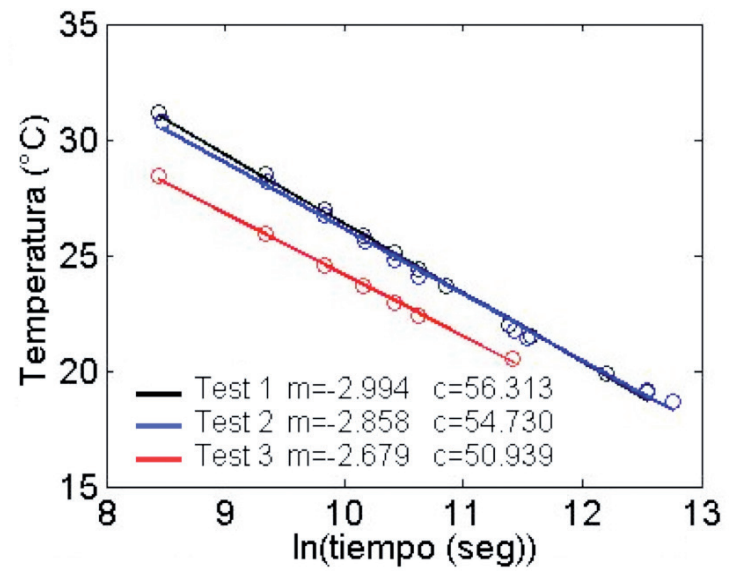

Figura 9: Ena) se muestran los datos y curvas de ajustes respectivos para 3 pruebas de enfriamiento: Tests 1, 2 y 3. La temperatura inicial de enfriamiento, para cada caso respectivamente, es de $38.4^{\circ} \mathrm{C}, 38.35^{\circ} \mathrm{C}$ y $35.8^{\circ} \mathrm{C}$. El eje horizontal corresponde al tiempo (horas) y en b) se muestran los mismos datos y curvas de a) en escala logarítmica en el eje del tiempo (segundos).

$$
q=\frac{2 \pi L\left(\mathrm{~T}_{1}-\mathrm{T}_{4}\right)}{\frac{1}{\lambda_{\mathrm{a}}} \ln \left(\frac{r_{2}}{r_{1}}\right)+\frac{1}{\lambda_{\mathrm{b}}} \ln \left(\frac{r_{3}}{r_{2}}\right)+\frac{1}{\lambda_{\mathrm{c}}} \ln \left(\frac{r_{4}}{r_{3}}\right)}
$$

La variable $q$ es el flujo de calor total transferido entre el fluido portador de calor y el terreno en W. Estimar $q$ nos permite, desde la ecuación (3), asociar la pendiente $m$ con la conductividad $\lambda$. $\lambda$ es estimado como una suma porcentual, según el espesor que involucra, de las conductividades de la tubería, el relleno y el terreno.

Aplicamos un test de Montecarlo y ajustamos cada modelo al conjunto de observaciones.

$\mathrm{T}_{1}$ es la temperatura media del pozo para cada prueba $\mathrm{y}$ la hacemos variar en el rango $\mathrm{T}_{1}=41$ a $35^{\circ} \mathrm{C}$; su mejor ajuste es $\mathrm{T}_{1}=40 \pm 1{ }^{\circ} \mathrm{C}$. $\mathrm{T}_{4}$ es la temperatura media del 
terreno sin perturbar y su ajuste corresponde a $\mathrm{T}_{4}=15 \pm$ $1{ }^{\circ} \mathrm{C}$. $L$ es el largo de la tubería; el ajuste requiere el valor que conocemos, $L=69.5 \pm 0.5 \mathrm{~m} . r_{1}, r_{2}, r_{3} \mathrm{y} r_{4}$ son el radio interno de la tubería $(=0.013 \mathrm{~m})$, radio externo de tubería $(=0.016 \mathrm{~m})$, radio del relleno $(=0.035 \mathrm{~m})$ y el radio hasta el punto del terreno de temperatura inalterada. Al dejar a $r_{4}$ como variable aleatoria $(0.3-0.9 \mathrm{~m})$, los mejores ajustes se corresponden con un valor de $r_{4}=0.5 \pm 0.05 \mathrm{~m}$.

La conductividad térmica de la tubería y el relleno del pozo, respectivamente, aunque las podemos asumir conocidas, al dejarlas como variables aleatorias (1.5 - 3 $\mathrm{Wm}^{-1} \mathrm{~K}^{-1}$ ) resultan al ajustar en $\lambda_{\mathrm{a}}=0.5 \pm 0.05 \mathrm{y} \lambda_{\mathrm{b}}=1.65$ $\pm 0.05 \mathrm{Wm}^{-1} \mathrm{~K}^{-1}$.

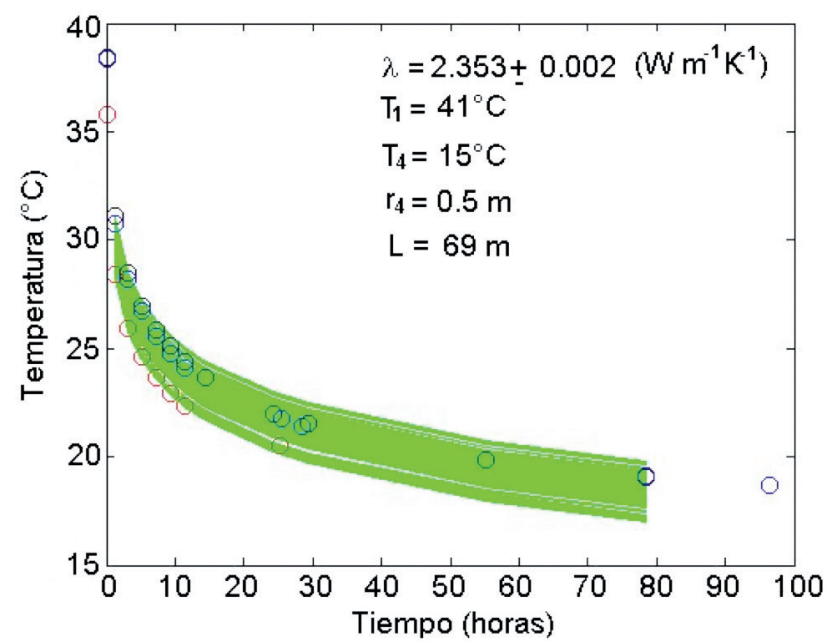

Figura 10: Se muestra el resultado de aplicar Test de Montecarlo. Cada curva de color verde es el resultado de un proceso de regresión lineal para valores de $\lambda_{\mathrm{c}}$ (por tanto, de $\lambda$ ). $L, \mathrm{~T}_{1}, \mathrm{~T}_{4} r_{4}$, $\mathrm{R}_{\mathrm{b}} \lambda_{\mathrm{a}}$ y $\lambda_{\mathrm{b}}$ son variables con menor sensibilidad respecto a la conductividad del terreno $\lambda_{\text {c }}$. Para los ajustes que se muestran, $\lambda_{a}$ $=0.5 \mathrm{Wm}^{-1} \mathrm{~K}^{-1}, \lambda_{\mathrm{b}}=1.64 \mathrm{Wm}^{-1} \mathrm{~K}^{-1} \mathrm{y} \mathrm{R}_{\mathrm{b}}=0.25 \mathrm{mKW}^{-1}$.

\section{Díscusión}

A través del ajuste numérico de un modelo de calentamiento TRT y/o enfriamiento (desde el apagado del TRT) de un pozo térmico vertical cerrado, buscamos determinar la conductividad térmica $\lambda$ más representativa para el sistema tubería, relleno y terreno, que constituye en el entorno del pozo. A partir del experimento TRT, el rango de valores encontrados para $\lambda$ es 1.6 a $2.5 \mathrm{Wm}^{-1} \mathrm{~K}^{-1}$, mientras que, usando los datos de enfriamiento del pozo, la conductividad térmica resulta ser exactamente $2.35 \mathrm{Wm}^{-}$ ${ }^{1} \mathrm{~K}^{-1}$. El ajuste realizado aquí entrega también un rango de valores para todas las variables en proceso, estableciendo que la conductividad térmica del terreno es altamente sensible, determinando así el valor indicado. Aunque para el sector no se cuenta con una estratigrafía, el rango de valores obtenidos coincide con aquellos asociados a zonas de arcilla, limo, arena, grava, conglomerados, propias del valle central chileno en la zona centro-sur.

\section{Conclusiones}

Podemos constatar que la temperatura del sub-suelo, por debajo de los $14 \mathrm{~m}$, se mantiene constante, con la peculiaridad de cumplir una regla más o menos general (según IGSHPA: Ground-Source Heat Pump Association): la temperatura del sub-suelo es igual al promedio anual de temperaturas en superficie más $1{ }^{\circ} \mathrm{C}$, es decir, $16.2^{\circ} \mathrm{C}$ aproximadamente.

El ajuste numérico basado en los modelos expuestos y sujeto a las mediciones de TRT así como la temperatura de enfriamiento del pozo geotérmico en estudio, resulta satisfactorio en el sentido de generar valores de conductividad térmica que bien se pueden asociar a un rango de posibilidades coincidentes con los expuestos en la literatura, según la constitución estratigráfica esperada para la zona: materiales como la arcilla o limo alcanzan los $1.5 \mathrm{Wm}^{-1} \mathrm{~K}^{-1}$, la arena y grava $2.0 \mathrm{Wm}^{-1} \mathrm{~K}^{-1}$, conglomerados $\mathrm{y}$ areniscas 1.5 a $2.0 \mathrm{Wm}^{-1} \mathrm{~K}^{-1}$. En el caso del presente estudio, este valor alcanza los $2.35 \mathrm{Wm}^{-1} \mathrm{~K}^{-1}$.

Respecto de la capacidad de enfriamiento del intercambiador de calor geotérmico, se puede constatar, por una parte, que un modelo 1-D (ley de conducción de Fourier), se ajusta bien a las observaciones, pudiendo además ratificar el orden de valores de conductividad térmica determinados a través del experimento de TRT. El modelo resulta ser altamente sensible a la variable conductividad térmica del terreno $\lambda_{\mathrm{c}}$, la que determina el valor final de $\lambda$ por sobre los valores de conductividad térmica de la tubería y el relleno.

\section{Referencias}

Carslaw, H.S. and Jaeger, J.C. (1959). Conduction of heat in solids. $2^{\text {nd }}$ ed., Oxford University Press, Oxford, UK

Castro, D., Pascual, P. y Indacoechea, I. (2012). Proyecto de aprovechamiento geotérmico de baja entalpía en edificio de usos múltiples. Parque Tecnológico. Tomo II: Campo de Sondas. Informe. Talca, Chile 
del Valle Fernández, P. (2012). Uso conjunto de diagrafías y TRT para la determinación de parámetros térmicos de un sondeo. Tesis de Máster en Ingeniería Energética, Universidad de Oviedo, España

Ingersoll, L.R., Zobel, O.J. and Ingersoll, A.C. (1954). Heat conduction with engineering, geological, and other applications. University of Wisconsin Press, USA

Jara Morales, L.S. y Martínez Martínez, M.V. (2016). Guía técnico-económica para la construcción de sistemas de climatización geotérmica aplicado a viviendas unifamiliares. Tesis de pregrado, Universidad Católica del Maule, Talca

Lee, C., You, J. and Park, H. (2018). In-situ response test of various borehole depths and heat injection rates at standing column well geothermal heat exchanger systems. Energy and Buildings 172, 201-208

Llopis Trillo, G. y Rodrigo Angulo, V. (2008). Guía de la energía geotérmica. Fundación de la Energía de la Comunidad de Madrid, España

Mogensen, P. (1983). Fluid to duct wall heat transfer in duct system heat storages. Proceedings of the International Conference on Subsurface Heat Storage in Theory and Practice, Stockholm, Sweden, 652-657
Monzó, P.M. (2011). Comparison of different Line Source Model approaches for analysis of thermal response Test in a U-pipe Borehole Heat Exchanger. MSc thesis, KTH School of Industrial Engineering and Management, Stockholm, Sweden

Natural Resources Canada (2004). Heating and cooling with a heat pump (EnerGuide). Office of Energy Efficiency, Energy Publications, Canada

Oyarce, C.N. (2018). Caracterización geotécnica de los suelos de fundación de la ciudad de Talca. Tesis de pregrado, Universidad Católica del Maule, Talca

Sharqawy, M.H., Said, S.A., Mokheimer, E.M., Habib, M.A., Badr, H.M. and Al-Shayea, N.A. (2009). First in situ determination of the ground thermal conductivity for boreholeheat exchanger applications in Saudi Arabia. Renewable Energy 34(10), 22182223

VDI 4640 (2010). Thermische Nutzung des Untergrundes Grundlagen, Genehmigungen, Umweltaspekte. Beuth Verlag, Berlin, Germany 